\title{
VIGILÂNCIA DO CRESCIMENTO E DESENVOLVIMENTO: ANÁLISE DOS REGISTROS NA CADERNETA DE SAÚDE DA CRIANÇA
}

\author{
Altamira Pereira da Silva Reichert ${ }^{1}$, Daniele de Souza Vieira ${ }^{2}$, Nathanielly Cristina Carvalho de Brito Santos ${ }^{3}$, \\ Tarciane Marinho Albuquerque ${ }^{4}$, Neusa Collet ${ }^{5}$, Elenice Maria Cecchetti Vaz ${ }^{6}$
}

RESUMO: Objetivou analisar o registro de dados sobre vigilância do crescimento e desenvolvimento de crianças menores de um ano na caderneta de saúde da criança. Estudo transversal com abordagem quantitativa. O material empírico foi coletado em 116 cadernetas, entre dezembro de 2012 e março de 2013, em João Pessoa - Paraíba. Para análise, utilizouse o teste qui-quadrado e o exato de Fisher. Nenhuma caderneta continha registros acerca da saúde bucal e do número do cartão do Sistema Único de Saúde, 109 (94\%) não apresentavam dados do pré-natal e 115 (99,1\%) não tinham informações referentes às ações da Primeira Semana Saúde Integral. Houve associação significativa entre o número de consultas e idade das crianças e dados antropométricos, com maior porcentagem de falhas nos registros daquelas que tiveram entre uma e seis consultas. Espera-se que os profissionais de saúde reflitam sobre seu processo de trabalho quanto à valorização da Caderneta de Saúde da Criança.

DESCRITORES: Saúde da criança; Crescimento e desenvolvimento; Atenção primária à saúde; Registros de saúde pessoal; Saúde da Família.

\section{GROWTH AND DEVELOPMENT SURVEILLANCE: ANALYSIS OF RECORDS IN THE CHILD HEALTH HANDBOOK}

ABSTRACT: The objective in this study was to analyze the records of growth and development data for children under one year of age in the child health handbook. Cross-sectional study with quantitative approach. The empirical material was collected in 116 handbooks between December 2012 and March 2013 in João Pessoa - Paraíba, Brazil. The chi-square test and Fisher's exact test were used for analysis. None of the handbooks contained oral health records and the number of the Unified Health System card, 109 (94\%) did not present prenatal data and $115(99.1 \%)$ did not contain information on the actions of the First Integral Health Week. A significant association was found between the number of consults and the children's age and anthropometric data, with a higher percentage of missing records for children who took part in one to six consults. The health professionals are expected to reflect on their work process regarding the valuation of the Child Health Handbook.

DESCRIPTORS: Child health; Growth and development; Primary health care; Health records, personal; Family health.

\section{VIGILANCIA DEL CRECIMIENTO Y DESARROLLO: ANÁLISIS DE LOS REGISTROS EN LA TARJETA DE SALUD DEL NIÑO}

RESUMEN: La finalidad del estudio fue analizar el registro de datos acerca de acompañamiento de crecimiento y desarrollo de niños de menos de un año por la tarjeta de salud de niños. Estudio transversal con abordaje cuantitativo, cuyo material empírico fue obtenido en 116 tarjetas, entre diciembre de 2012 y marzo de 2013, en João Pessoa - Paraíba. Para análisis, fue utilzado el test chi cuadrado y el exato de Fisher. Ninguna tarjeta contenía registros acerca de la salud bucal y del número en el Sistema Único de Salud; 109 (94\%) no presentaban datos del prenatal; y $115(99,1 \%)$ no traían informaciones de las acciones de la Primera Semana Salud Integral. Hubo significativa asociación entre el número de consultas y edad de los niños y datos antropométricos, con mayor porcentaje de fallas en los registros de aquellos que tuvieron entre una y seis consultas. Se espera que los profesionales de salud hagan una reflexión acerca de su proceso de trabajo cuanto a la valoración de la Tarjeta de Salud del Niño.

DESCRIPTORES: Salud del niño; Crecimiento y desarrollo; Atención primaria a la salud; Registros de salud personal; Salud de la Familia.

'Enfermeira. Doutora em Saúde da Criança e do Adolescente. Docente de Enfermagem e do Programa de Pós-Graduação em Enfermagem da Universidade Federal da Paraíba. João Pessoa, PB, Brasil.

${ }^{2}$ Enfermeira. Mestranda em Enfermagem. Universidade Federal da Paraíba. João Pessoa, PB, Brasil.

${ }^{3}$ Enfermeira. Doutoranda em Enfermagem. Docente de Enfermagem da Universidade Federal de Campina Grande. Cuité, PB, Brasil. ${ }^{4}$ Enfermeira. Mestranda do Programa de Pós-Graduação em Enfermagem da Universidade Federal da Paraíba. João Pessoa, PB, Brasil. ${ }^{5}$ Enfermeira. Doutora em Enfermagem. Docente de Enfermagem e do Programa de Pós-Graduação em Enfermagem da Universidade Federal da Paraíba. João Pessoa, PB, Brasil.

${ }^{6}$ Enfermeira. Doutoranda em Enfermagem. Docente de Enfermagem da Universidade Federal Fluminense. Niterói, RJ, Brasil.

Autor Correspondente:

Daniele de Souza Vieira

Universidade Federal da Paraíba

R. São Marcos, 206 - 58308-250 - Bayeux, PB, Brasil

E-mail: daniele.vieira2015@gmail.com
Recebido: 17/02/2016 Finalizado: 25/10/2016 


\section{- INTRODUÇÃO}

Existe um crescente reconhecimento na literatura de que a saúde nos primeiros anos estabelece as bases para o bem-estar ao longo da vida. Este ponto de vista é particularmente valioso para a compreensão do potencial das políticas e programas voltados para a primeira infância, sob o intuito de produzir benefícios à vida adulta(1). É importante, então, o enfoque na promoção da saúde e prevenção das doenças em crianças, a fim de proporcionar crescimento e desenvolvimento saudáveis.

Estima-se que a cada ano, no mundo, 6,6 milhões de crianças menores de cinco anos de idade morrem de complicações no período neonatal e de doenças comuns na infância que poderiam ser evitadas se houvesse melhores cuidados de saúde. Mesmo com progressos nas intervenções de saúde materna, neonatal e infantil nas últimas duas décadas, houve limitados avanços na melhoria dos resultados pediátricos devido a existência de lacunas entre a cobertura e a qualidade dos cuidados prestados nas unidades de saúde ${ }^{(2)}$.

Visando superar essas lacunas, no Brasil, o monitoramento das ações básicas de saúde foi instituído pelo Programa de Assistência Integral à Saúde da Criança (PAISC), após a constituição de 1988, por meio da utilização do Cartão da Criança $(C C)^{(3)}$, sendo posteriormente substituído pela Caderneta de Saúde da Criança (CSC), instrumento de direito infantil, essencial para vigilância à saúde até os 9 anos de idade. Esta contém informações e orientações relevantes para o cuidado integral, com distribuição gratuita e obrigatória em todo o território brasileiro(4).

A adequada utilização da CSC possibilita apropriação do instrumento pela família, cuja potencialidade para adesão e corresponsabilidade pelos cuidados desenvolvidos repercute positivamente na redução da morbimortalidade infantil(5). Ademais, propicia aos profissionais e familiares identificar precocemente qualquer alteração no crescimento e desenvolvimento infantil. Portanto, caracteriza-se como um instrumento de acompanhamento longitudinal e de educação em saúde, capaz de auxiliar na atenção integral à saúde da criança ${ }^{(6)}$.

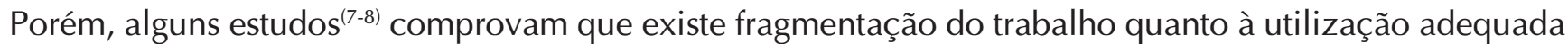
da CSC pelas Equipes de Saúde da Família, pois ações aparentemente simples como pesar, medir e avaliação neuropsicomotora nem sempre são registradas e, quando são, não estão sendo realizadas de forma correta e sistemática, comprometendo a eficácia de um importante instrumento que visa o acompanhamento integral da saúde na infância. Também não identificaram registros na caderneta quanto aos procedimentos realizados na consulta de puericultura ${ }^{(9)}$.

Tendo em vista a importância da CSC como uma ferramenta para a vigilância à saúde da criança e considerando a necessidade de investigar o registro das informações pelos profissionais, conforme preconizado pelo Ministério da Saúde, o presente estudo tem por objetivo analisar o registro de dados sobre vigilância do crescimento e desenvolvimento de crianças menores de um ano na Caderneta de Saúde da Criança.

\section{MÉTODO}

Estudo transversal com abordagem quantitativa, realizado no período de dezembro de 2012 a março de 2013, em Unidades de Saúde da Família do Distrito Sanitário III (DS-III), na cidade de João Pessoa - Paraíba, Nordeste do Brasil, cuja rede de atenção básica dispõe de 180 Equipes de Saúde da Família distribuídas territorialmente em cinco Distritos Sanitários, responsáveis pela assistência à saúde de 568.082 pessoas.

O DS-III possuía, na época da coleta de dados, 53 Equipes de Saúde da Família responsáveis pela cobertura de 90,5\% das famílias desta área, sendo 16 caracterizadas como unidades isoladas, com apenas uma equipe, e nove unidades integradas contemplando de três a quatro equipes na mesma estrutura física e organizacional.

A população do estudo foi composta por crianças menores de um ano atendidas nas Unidades de Saúde da Família (USF), representadas por meio de suas cadernetas de saúde. Foram incluídas na amostra as crianças com até um ano de idade e suas respectivas cadernetas de saúde. Foram excluídas crianças não cadastradas na USF Isolada do DS-III, que a mãe não portava a caderneta no momento da coleta de dados e as que utilizam o serviço de saúde de forma esporádica. 
Para compor a amostra do estudo considerou-se o número de crianças cadastradas nas USF isoladas, que no mês de julho de 2012 correspondia a 553 crianças. O cálculo amostral resultou em 228 CSC, adotando-se um erro tolerável de 5\% e Intervalo de Confiança de 95\%. Para seleção da amostra utilizou-se o método de amostragem não-probabilística por acessibilidade, com a coleta de dados realizada durante o comparecimento das mães às USF. No período da coleta de dados uma unidade de saúde estava desativada para reforma, o que fez com que a amostra final resultasse em 116 CSC.

A coleta foi realizada com um formulário para a verificação direta dos registros realizados na CSC, entre eles o preenchimento correto nas curvas de Perímetro Cefálico (PC), Peso (P), Comprimento (C) e Índice de Massa Corpórea (IMC), e no quadro de vigilância do desenvolvimento neuropsicomotor.

Para avaliação do preenchimento da CSC, utilizou-se como referência as orientações contidas no Manual para Utilização da Caderneta de Saúde da Criança do Ministério da Saúde ${ }^{(2)}$, sendo considerado adequado o registro que atendesse aos critérios do MS, e apenas como item preenchido ou não preenchido para aquelas informações sem critérios definidos pelo mesmo órgão.

Quanto aos dados presentes nas curvas do crescimento, admitiu-se pelo menos um registro das medidas antropométricas (PC, Peso, Comprimento/Estatura e IMC) com intervalo máximo de três meses, conforme o calendário mínimo de acompanhamento preconizado pelo Ministério da Saúde, ou seja, no mínimo sete consultas de puericultura até um ano de idade. No que concerne à avaliação do desenvolvimento neuropsicomotor, foi avaliada a presença de algum registro dos marcos do desenvolvimento para a faixa etária de idade na CSC.

Os dados foram digitados no programa Excel versão 2010 e analisados a partir do Software Statistical Package Social Science(SPSS), versão 20.0 for Windows. O estudo envolveu estatística descritiva representada pela frequência absoluta e relativa das variáveis, e foram estudadas possíveis associações entre as variáveis, idade da criança e número de consulta, utilizando o teste de Qui-quadrado para tabela de contingência $2 \times 2$. Quando as frequências observadas foram menores que cinco, considerou-se o teste exato de Fisher.

A pesquisa foi aprovada pelo Comitê de Ética em Pesquisa do Centro de Ciência da Saúde da Universidade Federal da Paraíba, sob protocolo n 0096/12. As mães/responsáveis das crianças foram esclarecidas sobre os objetivos e métodos da pesquisa e assinaram livremente o termo de consentimento.

\section{- RESUlTADOS}

O estudo evidencia que o preenchimento dos dados se mostrou precário, pois nenhuma CSC estava com todos os itens registrados devidamente. O único item presente em todas as CSC foi data de nascimento da criança, enquanto que o registro de procedimentos que envolvem a saúde bucal e o número do cartão do SUS não estava presente em nenhuma caderneta.

Entre as 116 cadernetas investigadas, $71(61,2 \%)$ das crianças tinham idades entre zero e seis meses, sugerindo que existe um cuidado maior à criança nesses primeiros meses. Segundo registros, 107 (92,2\%) das crianças realizou teste de triagem neonatal. Sete $(6 \%)$ das CSC continham informações acerca da realização de pré-natal, porém, as informações alusivas ao tipo e local de parto foram mais expressivas, com 42 (36,2\%) e $61(52,6 \%)$, respectivamente.

Em relação aos dados do nascimento, o percentual de preenchimento do peso ao nascer foi 106 (91,4\%), o que não ocorreu com as ações referentes à "Primeira Semana Saúde Integral" e o seguimento do recémnascido na unidade de saúde, com apenas um (0,9\%) dos registros presentes na caderneta (Tabela 1$)$.

Tabela 1 - Frequência dos registros avaliados nas Cadernetas de Saúde da Criança. João Pessoa, PB, Brasil, 2013 (continua)

\begin{tabular}{llll} 
Variáveis & & $\mathbf{n}$ & $\mathbf{\%}$ \\
\hline Das crianças & & & \\
\hline Idade das crianças & 0 a 6 meses & 71 & 61,2 \\
\cline { 2 - 4 } & 7 a 12 meses & 45 & 38,8 \\
\hline
\end{tabular}




\begin{tabular}{|c|c|c|c|}
\hline \multirow[t]{2}{*}{ Sexo } & Feminino & 55 & 47,4 \\
\hline & Masculino & 61 & 52,6 \\
\hline \multicolumn{4}{|l|}{ Dados identificação na CSC } \\
\hline \multirow[t]{2}{*}{ Nome da criança } & Sim & 101 & 87,1 \\
\hline & Não & 15 & 12,9 \\
\hline \multirow[t]{2}{*}{ Data de nascimento } & Sim & 116 & 100 \\
\hline & Não & - & - \\
\hline \multirow[t]{2}{*}{ Nome dos pais } & Sim & 108 & 93,1 \\
\hline & Não & 8 & 6,9 \\
\hline \multirow[t]{2}{*}{$\mathrm{N}$ do registro do prontuário } & Sim & 14 & 12,1 \\
\hline & Não & 102 & 87,9 \\
\hline \multirow[t]{2}{*}{ N da Declaração de nascido vivo } & Sim & 54 & 46,6 \\
\hline & Não & 62 & 53,4 \\
\hline \multirow[t]{2}{*}{ N do cartão do SUS } & Sim & - & - \\
\hline & Não & 116 & 100 \\
\hline \multirow[t]{2}{*}{ Dados do pré-natal } & Sim & 7 & 6 \\
\hline & Não & 109 & 94 \\
\hline \multicolumn{4}{|l|}{ Dados do Parto e pós-parto } \\
\hline \multirow[t]{2}{*}{ Local de parto } & Sim & 61 & 52,6 \\
\hline & Não & 55 & 47,4 \\
\hline \multirow[t]{2}{*}{ Tipo de parto } & Sim & 42 & 36,2 \\
\hline & Não & 74 & 63,8 \\
\hline \multicolumn{4}{|l|}{ Dados do Recém-nascido } \\
\hline \multirow[t]{2}{*}{ Peso ao nascer } & Sim & 106 & 91,4 \\
\hline & Não & 10 & 8,6 \\
\hline \multirow[t]{2}{*}{ Comprimento ao nascer } & Sim & 104 & 89,7 \\
\hline & Não & 12 & 10,3 \\
\hline \multirow[t]{2}{*}{ Perímetro cefálico } & Sim & 102 & 87,9 \\
\hline & Não & 14 & 12,1 \\
\hline \multirow[t]{2}{*}{ Índice de APGAR } & Sim & 102 & 87,9 \\
\hline & Não & 14 & 12,1 \\
\hline \multirow[t]{2}{*}{ Teste do pezinho } & Sim & 107 & 92,2 \\
\hline & Não & 9 & 7,8 \\
\hline \multirow[t]{2}{*}{ Procedimentos de Saúde bucal } & Sim & - & - \\
\hline & Não & 116 & 100 \\
\hline \multirow[t]{2}{*}{ Registro de Intercorrências Clínicas } & Sim & 2 & 1,7 \\
\hline & Não & 114 & 98,3 \\
\hline \multirow[t]{2}{*}{ Registro quanto à Primeira Semana } & Sim & 1 & 0,9 \\
\hline & Não & 115 & 99,1 \\
\hline \multirow[t]{3}{*}{ Registro de consultas realizadas } & Uma a seis & 85 & 73,3 \\
\hline & Sete ou mais & 10 & 8,6 \\
\hline & Nenhuma & 21 & 18,1 \\
\hline
\end{tabular}

Na Tabela 2 pode-se observar o comprometimento de registros sobre a avaliação antropométrica, visto que apenas $40(34,5 \%)$ das cadernetas apresentaram registro na curva do peso para a idade em número preconizado pelo MS, da mesma forma, apenas $22(19 \%)$ dessas possuía registro do comprimento para a idade. Quanto ao Perímetro Cefálico, constatou-se 66 (56,9\%) dos registros não atendiam aos requisitos para 
serem considerados adequados e em $113(97,4 \%)$ das cadernetas não foram encontrados registros do IMC. Em relação ao desenvolvimento neuropsicomotor infantil, 36 (31\%) das cadernetas tinham registrados algum marco do desenvolvimento para faixa etária.

Tabela 2 - Frequência dos registros de crescimento e desenvolvimento infantil nas Cadernetas de Saúde da Criança. João Pessoa, PB, Brasil, 2013

\begin{tabular}{lll} 
Variáveis & $\mathbf{n}$ & $\mathbf{\%}$ \\
\hline Dados acerca do Desenvolvimento Infantil & & \\
\hline Sim & 36 & 31 \\
\hline Não & 80 & 69 \\
\hline Perímetro Cefálico & & \\
\hline Sim & 50 & 43,1 \\
\hline Não & 66 & 56,9 \\
\hline Registro no gráfico Peso/Idade & & \\
\hline Sim & 40 & 34,5 \\
\hline Não & 76 & 65,5 \\
\hline Registro no gráfico comprimento/Idade & & 19 \\
\hline Sim & 22 & 81 \\
\hline Não & 94 & \\
\hline Registro do IMC no gráfico & & 2,6 \\
\hline Sim & 3 & 97,4
\end{tabular}

Neste estudo foi possível identificar associação estatisticamente significativa entre a idade da criança e a frequência de consultas registradas, $p=0,000$, isso porque o número de consultas foi expressivamente maior em crianças com idade inferior a seis meses. Todavia, apesar da frequência maior de consultas nesta faixa etária, não se observou um quantitativo de registro de avaliações antropométricas adequadas, pois houve uma relação estatisticamente significativa entre a diminuição dos registros antropométricos corretos em crianças que realizaram até seis consultas (Tabela 3).

Tabela 3 - Relação entre o número de consultas e as variáveis idade das crianças e dados antropométricos registrados na Caderneta de Saúde da Criança. João Pessoa, Paraíba, Brasil, 2013 (n=95)

\begin{tabular}{|c|c|c|c|c|c|}
\hline & \multicolumn{5}{|c|}{ Consultas } \\
\hline & \multicolumn{2}{|c|}{ De 1 a 6* } & \multicolumn{2}{|c|}{7 ou mais } & \multirow[b]{2}{*}{ Valor de $\mathrm{p}$} \\
\hline & $\mathbf{n}$ & $\%$ & $\mathbf{n}$ & $\%$ & \\
\hline \multicolumn{6}{|c|}{ Idade das crianças } \\
\hline $0-6$ meses & 50 & 58,8 & - & - & $0,000^{* *}$ \\
\hline $7-12$ meses & 35 & 41,2 & 10 & 100 & - \\
\hline \multicolumn{6}{|c|}{ Dados antropométricos } \\
\hline Correto & 11 & 12,9 & 7 & 70 & - \\
\hline Incorreto & 74 & 87,1 & 3 & 30 & $0,000^{* *}$ \\
\hline
\end{tabular}

*O Ministério da Saúde preconiza que 7 ou mais consultas são consideradas o quantitativo ideal para crianças de até um ano de idade.

** Teste Exato de Fisher - resultado significativo, $p<0,05$. 


\section{- DISCUSSÃO}

A Caderneta de Saúde da Criança é um instrumento fundamental para a vigilância do crescimento e desenvolvimento infantil. Porém, o presente estudo revela fragilidade no registro das informações antes mesmo do nascimento da criança, pois os dados referentes ao pré-natal não foram registrados na maioria das cadernetas. Isso pode repercutir diretamente na saúde de recém-nascidos, uma vez que a ausência de registro poderá comprometer a comunicação entre profissionais que acompanharam a mãe e aqueles que darão o seguimento da criança na puericultura.

Os dados referentes ao pré-natal deveriam estar registrados em todas as cadernetas, pois a exposição da mãe a algumas substâncias deve ser comunicada e registrada, tendo em vista que podem resultar em anormalidades na criança ${ }^{(10)}$.

A "Primeira Semana Saúde Integral", ação preconizada pelo Ministério da Saúde na Agenda de Compromissos para a Saúde Integral da Criança e Redução da Mortalidade Infantil ${ }^{(11)}$, é caracterizada como uma estratégia que prioriza a atenção à saúde da mulher e ao recém-nascido nas unidades básicas de saúde, sob o intuito de proporcionar a promoção dos cuidados de saúde e identificação de riscos de agravos à criança $^{(12)}$, como também o esclarecimento de dúvidas e correções de práticas inadequadas por parte da mãe/família.

Apesar de sua importância para a redução da morbimortalidade infantil, observa-se que o tema não tem sido abordado em estudos relacionados a CSC e nem nos que abordam registros dos profissionais, o que pode resultar em falta de orientações às mães para esta estratégia ainda no pré-natal. Considera-se esse tema relevante, pois o baixo índice de registro dessas ações nas cadernetas marca a descontinuidade da assistência, comprometendo a procura à unidade de saúde pelas puérperas em um momento de muitas demandas advindas do período pós-parto.

Em consonância com outros estudos, o registro do peso ao nascer foi realizado de forma satisfatória( ${ }^{(7,13)}$. Isso pode ser justificado por se tratar de uma das informações mais requeridas do recém-nascido, tanto pela sua família quanto pelos profissionais; para esses, o peso ao nascer é um dado fundamental, tendo em vista que algumas doenças que acometem os adultos estão relacionadas às alterações no crescimento e desenvolvimentos intrauterinos, a exemplo das doenças cardiovasculares, metabólicas e endócrinas na vida adulta $^{(14)}$.

Um dado que merece destaque é o aumento dos registros relativos ao APGAR, principalmente quando comparados aos resultados encontrados em outros estudos realizados no Brasil, que descrevem ausência da anotação desse índice em cerca $40 \%$ das cadernetas $^{(7,13)}$. O teste de APGAR é um importante indicador da condição física do recém-nascido realizado no $1^{\circ}$, $5^{\circ}$ e $10^{\circ}$ minutos após o nascimento ${ }^{(15)}$. É um método simples e de suma importância para o acompanhamento de saúde da criança, pois os registros dessa informação possibilita aos profissionais de saúde a identificação de alguma intercorrência no nascimento.

Uma informação que merece preocupação é a ausência de registros referentes aos procedimentos de saúde bucal, apesar de essas orientações serem preconizadas para o acompanhamento da criança ${ }^{(16)}$. Não se sabe se essas ações não estão sendo realizadas na rotina da equipe multiprofissional, ou se os profissionais as fazem e não registram.

O panorama dos problemas de saúde bucal é persistente em muitas comunidades ao redor do mundo, especialmente entre os grupos menos favorecidos que vivem em países desenvolvidos e em desenvolvimento, como no Brasil. Os fatores comportamentais e sócio-ambientais que envolvem tais problemas são evidenciados em levantamentos epidemiológicos, os quais revelam que a carga global das condições bucais no período de 1990-2010 afetou cerca de 3,9 bilhões de pessoas em todo o mundo. A cárie dentária, por exemplo, ainda é a condição mais preocupante em países industrializados, atingindo cerca de $90 \%$ de crianças e a grande maioria dos adultos ${ }^{(17)}$. Sendo assim, a ausência desses registros e de orientações às mães demonstram a falta de atenção para tais problemas, que inviabilizam o acompanhamento de marcos importantes, como a erupção dentária, bem como a prevenção de cáries e outras doenças bucais que comprometem à saúde da criança.

Quanto aos registros dos marcos do desenvolvimento neuropsicomotor, os dados apontaram fragilidade no preenchimento com ausência de anotações numa quantidade expressiva de cadernetas. Esses dados 
vêm ao encontro de outro estudo que apresentou resultado semelhante, comprometendo a vigilância do desenvolvimento infanti|(8).

Corroborando esse aspecto, estudos ${ }^{(6,18)}$ afirmam que esta realidade repercute no déficit da vigilância do desenvolvimento infantil, limitando as possibilidades de prevenção e detecção precoce de algum atraso no desenvolvimento e, portanto, qualquer intervenção em tempo hábil. Essa atitude contrapõe-se às propostas do Ministério da Saúde para garantia da integralidade da atenção à saúde da criança, cujo acompanhamento deve ser cauteloso e contínuo, visto os intensos processos neuropsicomotores ocorridos em um curto período de tempo, e as negativas repercussões para a vida, em caso de alterações no desenvolvimento. Outrossim, para a promoção do desenvolvimento saudável é fundamental a educação em saúde e o incentivo ao estímulo do desenvolvimento neuropsicomotor da criança no ambiente domiciliar ${ }^{(19)}$.

Dentre os dados antropométricos, identificou-se que as curvas do perímetro cefálico e do peso destacaramse quanto ao preenchimento. Isso leva a inferir que pode estar ocorrendo um maior envolvimento dos profissionais para detecção precoce de alterações no desenvolvimento e identificação de fatores associados, sob o intuito de prevenir deficiências na vida adulta ${ }^{(20)}$. Estes achados estão divergentes do encontrado em outro estudo envolvendo a temática, no qual a curva do peso para a idade foi a mais utilizada, quando comparado com a do perímetro cefálico ${ }^{(21)}$.

Quanto ao comprimento para idade, identificou-se baixo percentual de preenchimento e o gráfico do IMC raramente foi utilizado, situação semelhante a outros estudos ${ }^{(8,21)}$. Esse déficit de registros é preocupante, pois sugere que os profissionais de saúde não estão valorizando o acompanhamento do crescimento infantil.

Uma avaliação correta do crescimento deve contemplar os registros de cada um dos marcadores pônderoestaturais, para antecipar a identificação precoce de alterações e possibilitar intervenção oportuna à criança. Contudo, ações específicas importantes para o acompanhamento do crescimento das crianças vêm sendo implementadas com pouca frequência durante as consultas, inexistindo o acompanhamento integral da criança e, por extensão, ausência dos registros importantes para a vigilância do crescimento na CSC ${ }^{(22)}$.

A associação estatisticamente significativa entre a idade da criança e a frequência de consultas $(p=0,000)$ realizadas nos primeiros seis meses de vida sugere que os profissionais estão mais cautelosos para o acompanhamento das crianças nos primeiros meses, reduzindo esse acompanhamento à medida que a criança vai crescendo. Esses achados estão condizentes com o que é preconizado pelo Ministério da Saúde, que indica sete consultas durante o primeiro ano de vida. Em contraposição, estudo ${ }^{(23)}$ realizado em outro estado do Nordeste diverge desses resultados, uma vez que o percentual de registros das consultas com crianças menores de seis meses ainda é baixo $(38,6 \%)$.

Os resultados também revelam uma associação estatisticamente significativa entre o registro antropométrico e o número de consultas $(p=0,000)$, indicando haver maior percentual de registros incorretos entre as crianças que realizaram de uma a seis consultas, entre as quais podemos destacar as crianças menores de seis meses de vida com maior porcentagem de consultas adequadas para idade. Isso pode estar relacionado ao fato do segmento do crescimento e desenvolvimento nesta fase ocorrer em intervalos menores, visto as singularidades na velocidade de crescimento do lactente, o que exige um olhar minucioso para identificação de fatores que possam interferir nesse processo.

No entanto, o aumento na frequência das consultas possivelmente está associado ao aumento da demanda de trabalho, que pode comprometer a efetividade dos registros nas consultas ${ }^{(24)}$. Tal realidade é preocupante, pois, pelo fato de o número de consultas ser um indicador de vigilância do crescimento e desenvolvimento infantil, lacunas existentes nos registros desses dados na caderneta comprometem o seguimento da criança pelo profissional de saúde e pela mãe e/ou cuidador.

Partindo da premissa que os registros na caderneta possibilitam a comunicação entre os profissionais de saúde e familiares, e entre profissionais de saúde de diferentes serviços, a articulação entre os membros da equipe e a valorização desse documento pelos familiares, devem se intensificar, buscando-se efetivar seu papel fundamental de vigilância do crescimento e desenvolvimento infantil(25).

A principal limitação deste estudo versa em torno da faixa etária escolhida para compor a amostra, que não permitiu visualizar a realidade das cadernetas de todas as crianças usuárias dos serviços participantes do estudo. 
A informação em saúde é de fundamental importância para a continuidade do cuidado. Porém, na contramão dessa assertiva, os resultados deste estudo evidenciam fragilidades no registro dos dados na Caderneta de Saúde da Criança no primeiro ano de vida. Assim sendo, a caderneta não poderá ser utilizada como ferramenta dialógica entre familiares e profissionais, tampouco as anotações serão compartilhadas entre os profissionais nos diferentes níveis de atenção. Esta situação marca uma forte ruptura na linha de cuidado para atenção integral à saúde da criança e vigilância do crescimento e desenvolvimento infantil.

Espera-se que os resultados desta pesquisa e as reflexões realizadas contribuam para que os profissionais de saúde reflitam sobre seu processo de trabalho quanto à valorização da Caderneta de Saúde da Criança como ferramenta indispensável para a promoção da saúde infantil. Além disso, é importante que os gestores reconheçam a necessidade de capacitações para os profissionais de saúde que atendem à criança, como forma qualificar o cuidado à criança menor de um ano na APS.

Sugere-se o desenvolvimento de outros estudos que busquem evidenciar os motivos pelos quais os profissionais não registram os dados corretamente na caderneta, conforme recomendação do Ministério da Saúde.

\section{- REFERÊNCIAS}

1. Mistry KB, Minkovitz CS, Riley AW, Johnson SB, Grason HA, Dubay LC, et al. A new framework for childhood health promotion: the role of policies and programs in building capacity and foundations of early childhood health. Am J Publ Health. 2012;102(9):1688-96.

2. World Health Organization and Partnership for Maternal, Newborn and Child Health. Consultation on improving measurement of the quality of maternal, newborn and child care in health facilities. Geneva: World Health Organization; 2013.

3. Ministério da Saúde (BR). Secretaria de Atenção à Saúde. Área Técnica de Saúde da Criança e Aleitamento Materno. Gestões e gestores de políticas de atenção à saúde da criança: 70 anos de história. Brasília: Ministério da Saúde; 2011.

4. Ministério da Saúde (BR). Secretaria de Atenção à Saúde. Área Técnica de Saúde da Criança e Aleitamento Materno. Manual para utilização da Caderneta de Saúde da Criança. Brasília: Ministério da Saúde; 2005.

5. Vieira JRB, Vieira FLD. Análise crítica da caderneta de saúde da criança como instrumento de educação Fonoaudiológica. Conhecimento Divers. [Internet] 2012;4(7) [acesso em 16 set 2014]. Disponível: http://www.revistas.unilasalle.edu.br/ index.php/conhecimento_diversidade/article/view/624/484.

6. Reichert APS, de Almeida AB, de Souza LC, Silva MEA, Collet N. Vigilância do crescimento infantil: conhecimento e práticas de enfermeiros da atenção primária à saúde. Rev. Rene. [Internet] 2012;13(1) [acesso em 17 abr 2014]. Disponível: http://www.revistarene.ufc.br/revista/index.php/revista/article/view/23/19.

7. Modes PSSA, Gaíva MAM. Users' satisfaction concerning the care delivered to children at primary healthcare services. Esc. Anna Nery. 2013;17(3):455-65.

8. Palombo CNT, Duarte LS, Fujimori E, Toriyama ATM. Use and records of child health handbook focused on growth and development. Rev. esc. enferm. USP. 2014;48(n.esp):60-7.

9. Malaquias TSM, Baldissera VAD, Higarashi IH. Percepções da equipe de saúde e de familiares sobre a consulta de puericultura. Cogitare Enferm. 2015;20(2):368-75.

10. Grandjean P. Prenatal prevention. Science. 2014;345(6203):1462.

11. Ministério da Saúde (BR). Secretaria de Atenção à Saúde. Área Técnica de Saúde da Criança e Aleitamento Materno. Agenda de compromisso para a saúde integral da criança e redução da mortalidade infantil. Brasília: Ministério da Saúde; 2005.

12. Souza MHN, Gomes TNC, Paz EPA, Trindade CS, Veras RCC. Estratégia acolhimento mãe-bebê: aspectos relacionados 
à clientela atendida em uma unidade básica de saúde do município do Rio de Janeiro. Esc. Anna Nery. 2011;15(4):671-7.

13. Faria M, Nogueira TA. Avaliação do uso da caderneta de saúde da criança nas unidades básicas de saúde em um município de Minas Gerais. Rev. Bras. Ciên. Saúde. 2013;11(38):8-15.

14. Shayeb AG, Harrild K, Bhattacharya S. Birth weight and ovulatory dysfunction. BJOG. 2014;121(3):281-9.

15. Persson M, Johansson S, Villamor E, Cnattingius S. Maternal overweight and obesity and risk of severe birthasphyxia-related complications in term infants: a population-based cohort study in Sweden. PLoS Medicine. [Internet] 2014;11(5):e1001648 [acesso em 15 jun 2015]. Disponível: http://dx.doi.org/ 10.1371/journal.pmed.1001648.

16. Ministério da Saúde (MS). Secretaria de Atenção à Saúde. Departamento de Atenção Básica. Saúde Bucal. Caderno de Atenção Básica, n 17. Brasília: Ministério da Saúde; 2008.

17. Laganà G, Abazi Y, Nastasi EB, Vinjolli F, Fabi F, Divizia M, et al. Oral health conditions in an Albanian adolescent population: an epidemiological study. BMC Oral Health. 2015;(15):67.

18. WHO Multicentre Growth Reference Study Group. WHO Child Growth Standards: Growth velocity based on weight, length and head circumference: Methods and development. Geneva: World Health Organization; 2009.

19. Pereira MM, da Penha TP, Vieira DS, Vaz EMC, Santos NCCB, Reichert APS. Prática educativa de enfermeiras na atenção primária à saúde, para o desenvolvimento infantil saudável. Cogitare Enferm. 2015;20(4):767-74.

20. Chattopadhyay N, Mitra K. Neurodevelopmental outcome of high risk newborns discharged from special care baby units in a rural district in India. J Public Health Res. 2015;4(1):7-12.

21. Linhares AO, Gigante DP, Bender E, Cesar JA. Avaliação dos registros e opinião das mães sobre a caderneta de saúde da criança em unidades básicas de saúde, Pelotas, RS. Rev. AMRIGS. [Internet] 2012;56(3) [acesso em 10 ago 2014]. Disponível: http://www.amrigs.com.br/revista/56-03/avaliacao\%20dos\%20registros.pdf.

22. Oliveira FFS, de Oliveira ASS, Lima LHO, Marques MB, Felipe GF, Sena IVO. Child care consultations held by nurses within the family health strategy. Rev. Rene. 2013;14(4):694-703.

23. Cunha CLF, da Silva RA, Gama MEA, Costa GRC, Costa ASV, Tonial SR. O uso de serviços de atenção primária à saúde pela população infantil em um estado do nordeste brasileiro. Cad. saúde colet. [Internet] 2013;21(2) [acesso em 26 nov 2014]. Disponível: http://dx.doi.org/10.1590/S1414-462X2013000200003.

24. Lin $\mathrm{H}$. Revisiting the relationship between nurse staffing and quality of care in nursing homes: an instrumental variables approach. J Health Econ. 2014;(37):13-24.

25. Abreu TGT, Viana LS, Cunha CLF. Desafios na utilização da caderneta de saúde da criança: entre o real e o ideal. J Manag Prim Health Care. 2012;3(2):80-3. 